\title{
TRANSIENT PROBABILITIES FOR A SIMPLE BIRTH-DEATH-IMMIGRATION PROCESS UNDER THE INFLUENCE OF TOTAL CATASTROPHES
}

\author{
RANDALL J. SWIFT
}

(Received 27 August 2000)

\begin{abstract}
The transient probabilities for a simple birth-death-immigration process are considered. Catastrophes occur at a constant rate, and when they occur, reduce the population to size zero.
\end{abstract}

2000 Mathematics Subject Classification. Primary 60J80.

1. Introduction. In this note, a simple birth-death-immigration process is considered, which is influenced by total catastrophes which, when they occur, reduce the population size to zero. Population processes under the influence of various types of catastrophes have been studied by Bartoszynski et al. [2], Brockwell et al. [3], and Kyriakidis [4, 5].

The process is formulated by letting $N(t)$ represent the size of the population at time $t$ and

$$
P_{n}(t)=P[N(t)=n \mid N(0)=0] .
$$

As in the simple birth-death process, births and deaths occur proportional to the population size with a birth rate $\lambda>0$ and a death rate $\mu>0$. Immigration will occur independent of population size with rate $\alpha>0$. Further, the occurrence of a catastrophe is also independent of population size and will occur at a rate $\gamma>0$. Thus, the process can be described by the following transition rates:

\begin{tabular}{lcc}
\hline Transition & Rate & \\
\hline$i \rightarrow i+1$ & $\lambda i+\alpha$ & $(i \geq 0)$ \\
\hline$i \rightarrow i-1$ & $\mu i$, & $(i \geq 1)$ \\
\hline$i \rightarrow 0$ & $\gamma$ & $(i \geq 1)$ \\
\hline
\end{tabular}

The special case of $\gamma=1$ was recently considered by Kyriakidis [4], who obtained the stationary probabilities for this process. The transient probabilities of a simple immigration-catastrophe process, where $\lambda=0$ and $\mu=0$, was obtained by Swift [6]. In the next section, the transient probabilities for the general case are derived. 
2. The transient probabilities. The standard argument using the forward Kolmogorov equations shows that $P_{n}(t)$ satisfies

$$
\begin{gathered}
P_{n}^{\prime}(t)=(n+1) \mu P_{n+1}(t)+((n-1) \lambda+\alpha) P_{n-1}(t)-(n(\mu+\lambda)+\alpha+\gamma) P_{n}(t), \\
P_{0}^{\prime}(t)=\gamma \sum_{i=1}^{\infty} P_{i}(t)+\mu P_{1}(t)-\alpha P_{0}(t) .
\end{gathered}
$$

Now

$$
\sum_{i=1}^{\infty} P_{i}(t)=1-P_{0}(t)
$$

so that

$$
P_{0}^{\prime}(t)=\gamma+\mu P_{1}(t)-(\alpha+\gamma) P_{0}(t)
$$

Letting

$$
\psi(s, t)=\sum_{k=0}^{\infty} P_{k}(t) s^{k}
$$

be the probability generating function (PGF) for the system, it follows from the standard generating function method, that $\psi(s, t)$ satisfies the partial differential equation

$$
\frac{\partial \psi(s, t)}{\partial t}=(\lambda s-\mu)(s-1) \frac{\partial \psi(s, t)}{\partial s}+\alpha(s-1) \psi(s, t)+\gamma(1-\psi(s, t)) .
$$

Since we are considering a process with immigrations, we can assume, for the sake of simplicity, the initial condition $P_{0}(0)=1$. This gives $\psi(s, 0)=1$.

As in most birth-death processes, the solution of the partial differential equation (2.6) for $\psi(\cdot, \cdot)$ depends upon the values of the parameters $\lambda$ and $\mu$. The PGF $\psi(\cdot, \cdot)$ is given below for three important cases. The method of solution of (2.6) is standard and indeed, it is interesting to note that these solutions can also be obtained using a computer algebra system such as Mathematica.

CASE 2.1 $(\lambda \neq \mu$ and $\lambda \neq 0)$. The PDE (2.6) has solution

$$
\begin{aligned}
\psi(s, t)= & \left.\frac{1}{(\alpha+\gamma) \lambda-\alpha \mu}\right) \\
\times & {\left[\gamma \lambda\left(\frac{(1-s) \lambda}{\lambda-\mu}\right)^{\gamma /(\lambda-\mu)}{ }_{2} F_{1}\left(\frac{\alpha \lambda+\gamma \lambda-\alpha \mu}{\lambda^{2}-\lambda \mu}, \frac{\lambda-\mu+\gamma}{\lambda-\mu}, \frac{(\lambda+\alpha)(\lambda-\mu)+\gamma \lambda}{\lambda(\lambda-\mu)}, \frac{\lambda s-\mu}{\lambda-\mu}\right)\right.} \\
& +(s-1)^{\gamma /(\lambda-\mu)}(\lambda s-\mu)^{-\alpha / \lambda+\gamma /(\mu-\lambda)}\left(\frac{(\mu-\lambda)(\lambda s-\mu)}{\mu-\lambda s+\lambda(s-1) e^{(\lambda-\mu) t}}\right)^{\alpha / \lambda+\gamma /(\lambda-\mu)} \\
& \times\left(\frac{(\mu-\lambda)(s-1)}{\lambda(s-1)+(\mu-\lambda s) e^{-(\lambda-\mu) t}}\right)^{-\gamma /(\lambda-\mu)} \\
& \times\left(((\alpha+\gamma) \lambda-\alpha \mu)-\gamma \lambda\left(\frac{\lambda}{\lambda(s-1)+(\mu-\lambda s) e^{-(\lambda-\mu) t}}\right)^{\gamma /(\lambda-\mu)}\right. \\
& \left.\left.\quad \times{ }_{2} F_{1}\left(\frac{\alpha \lambda+\gamma \lambda-\alpha \mu}{\lambda^{2}-\lambda \mu}, \frac{\lambda-\mu+\gamma}{\lambda-\mu}, \frac{(\lambda+\alpha)(\lambda-\mu)+\gamma \lambda}{\lambda(\lambda-\mu)},(\mu-\lambda s) e^{-(\lambda-\mu) t}\right)\right)\right],
\end{aligned}
$$


where ${ }_{2} F_{1}(a, b ; c, z)$ is the hypergeometric function defined by

$$
{ }_{2} F_{1}(a, b ; c, z)=\sum_{k=0}^{\infty} \frac{(a)_{k}(b)_{k}}{(c)_{k}} \frac{z^{k}}{k !}
$$

(cf. Agarwal [1]).

CASE $2.2(\lambda=\mu$ and $\lambda \neq 0)$. In this case, the PDE (2.6) has solution

$$
\begin{aligned}
\psi(s, t)= & \frac{\gamma e^{\gamma / \lambda(1-s)}}{\lambda(1-s)} \int_{1}^{\infty} z^{-\alpha / \lambda} e^{-\gamma z / \lambda(1-s)} d z-\frac{e^{-\gamma t}}{\lambda(1-s)}\left(\frac{1}{1+(1-s) t \lambda}\right)^{\alpha / \lambda} \\
& \times\left(\lambda(s-1)+\gamma(1+\gamma \lambda t(1-s)) \int_{1}^{\infty} z^{-\alpha / \lambda} e^{-\gamma(t+1 / \lambda(1-s)) z} d z\right) .
\end{aligned}
$$

CASE $2.3(\lambda=0$ and $\mu \neq 0)$. In this case, the PGF is given as

$$
\begin{aligned}
\psi(s, t)=\frac{1}{\mu}\left[e ^ { - \alpha s / \mu } \left(\gamma e^{\alpha / \mu} \int_{1}^{\infty} z^{-(1+\gamma / \mu)} e^{-\alpha(1-s) z / \mu} d z\right.\right. & \\
& \left.\left.+e^{-\gamma t}\left(\mu e^{\alpha\left(1-(1-s) e^{\mu t}\right) / \mu}-\gamma e^{\alpha / \mu} \int_{1}^{\infty} z^{-(1+\gamma / \mu)} e^{-\alpha(1-s) e^{\mu t} / \mu} d z\right)\right)\right] .
\end{aligned}
$$

Using the PGF, the probability of extinction, $P_{0}(t)$, can be obtained in each of the above cases.

CASE $2.1(\lambda \neq \mu$ and $\lambda \neq 0)$.

$$
\begin{aligned}
P_{0}(t)=\psi(0, t) & =\frac{1}{(\alpha+\gamma) \lambda-\alpha \mu}[ \\
& \times\left(\frac{\lambda-\mu}{\lambda e^{(\lambda-\mu) t}-\mu}\right)^{\alpha / \lambda+\gamma /(\lambda-\mu)}\left(\frac{\mu-\lambda}{\lambda-\mu e^{(-\lambda+\mu) t}}\right)^{\gamma /(\mu-\lambda)} \\
& \left.\quad{ }_{2} F_{1}\left(\frac{\alpha(\lambda-\mu)+\lambda \gamma}{\lambda(\lambda-\mu)}, \frac{\lambda-\mu+\gamma}{\lambda-\mu}, \frac{(\lambda+\alpha)(\lambda-\mu)+\gamma \lambda}{\lambda(\lambda-\mu)}, \frac{\mu}{\mu-\lambda e^{(\lambda-\mu) t}}\right)\right) \\
+\gamma \lambda\left(\frac{\lambda}{\lambda-\mu}\right)^{\gamma /(\lambda-\mu)} & \\
& \left.\times{ }_{2} F_{1}\left(\frac{\alpha(\lambda-\mu)}{\lambda(\lambda-\mu)+\lambda \gamma}, \frac{\lambda-\mu+\gamma}{\lambda-\mu}, \frac{(\lambda+\alpha)(\lambda-\mu)+\gamma \lambda}{\lambda(\lambda-\mu)}, \frac{\mu}{\mu-\lambda}\right)\right] .
\end{aligned}
$$

CASE $2.2(\lambda=\mu$ and $\lambda \neq 0)$.

$$
\begin{aligned}
P_{0}(t)=e^{\gamma / \lambda}[ & \left(\frac{1}{1+\lambda t}\right)^{\alpha / \lambda}\left(e^{-\gamma(1+\lambda t) / \lambda}-\frac{1}{\lambda}\left((\gamma+\gamma \lambda t) \int_{1}^{\infty} z^{-\alpha / \lambda} e^{-\gamma(t+1 / \lambda) z} d z\right)\right) \\
& \left.+\frac{\gamma}{\lambda} \int_{1}^{\infty} z^{-\alpha / \lambda} e^{-(\gamma / \lambda) z} d z\right] .
\end{aligned}
$$


CASE $2.3(\lambda=0$ and $\mu \neq 0)$.

$$
\begin{aligned}
P_{0}(t)= & \frac{e^{-\gamma t}}{\mu}\left(\mu e^{-\alpha\left(e^{t \mu}-1\right) / \mu}-\gamma e^{\alpha / \mu} \int_{1}^{\infty} z^{-(\gamma+\mu) / \mu} e^{-\left(\alpha e^{t \mu} / \mu\right) z} d z\right) \\
& +\frac{\gamma e^{\alpha / \mu}}{\mu} \int_{1}^{\infty} z^{-(\gamma+\mu) / \mu} e^{-(\alpha / \mu) z} d z .
\end{aligned}
$$

We note here that these expressions for $P_{0}(t)$, as $t \rightarrow \infty$, with $\gamma=1$, reduce to the stationary probabilities obtained by Kyriakidis.

The probabilities $P_{n}(t)$, for $n \geq 1$ can be obtained by expanding $\psi(s, t)$ as a power series in $s$. However, the nature of the representations (2.7), (2.9), and (2.10) for $\psi(s, t)$ makes this a formidable task. Alternatively, $P_{0}(t)$ can be used in (2.4) to obtain $P_{1}(t)$ then (2.1) can be used recursively, to obtain $P_{n}(t)$ for $n \geq 1$.

\section{REFERENCES}

[1] R. P. Agarwal, Generalized Hypergeometric Series, Uttar Pradesh Scientific Research Committee, Allahabad, India, Asia Publishing House, Bombay, 1963. MR 31\#3636.

[2] R. Bartoszynski, W. J. Buehler, W. Chan, and D. K. Pearl, Population processes under the influence of disasters occurring independently of population size, J. Math. Biol. 27 (1989), no. 2, 167-178. MR 90h:92017. Zbl 715.92022.

[3] P. J. Brockwell, J. Gani, and S. I. Resnick, Birth, immigration and catastrophe processes, Adv. in Appl. Probab. 14 (1982), no. 4, 709-731. MR 84d:60107. Zbl 496.92007.

[4] E. G. Kyriakidis, Stationary probabilities for a simple immigration-birth-death process under the influence of total catastrophes, Statist. Probab. Lett. 20 (1994), no. 3, 239-240. CMP 1294 110. Zbl 801.60073.

[5] E. G. Kyriakidis and A. Abakuks, Optimal pest control through catastrophes, J. Appl. Probab. 26 (1989), no. 4, 873-879. MR 90m:92066. Zbl 688.60069.

[6] R. J. Swift, A simple immigration-catastrophe process, Math. Sci. 25 (2000), no. 1, 32-36. CMP 1771175.

RANDAll J. Swift: Department of MAthematics, Western Kentucky University, BOWLING GREEN, KY 42101, USA

E-mail address: randa11. swi ft@wku .edu 


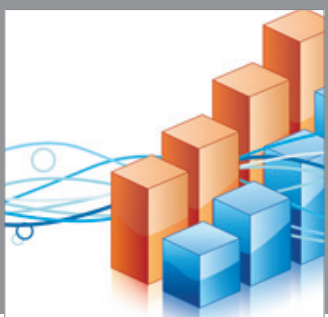

Advances in

Operations Research

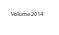

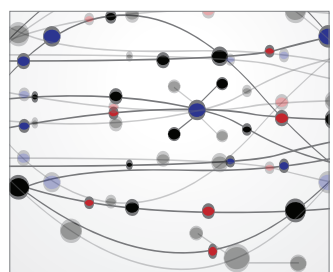

\section{The Scientific} World Journal
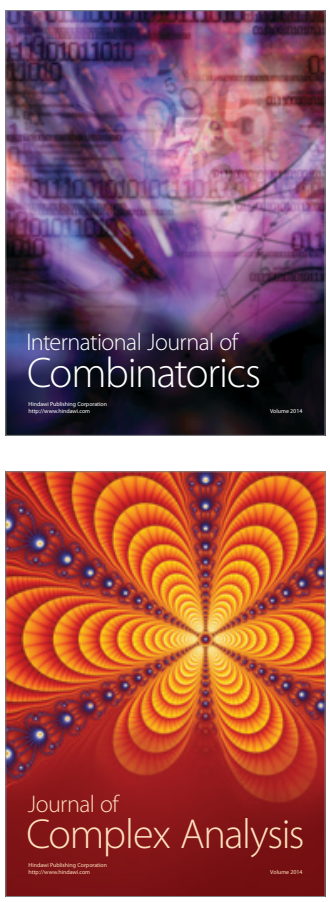

International Journal of

Mathematics and

Mathematical

Sciences
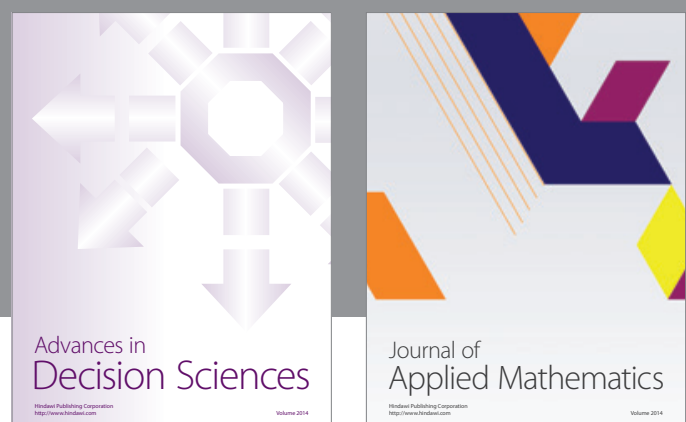

Journal of

Applied Mathematics
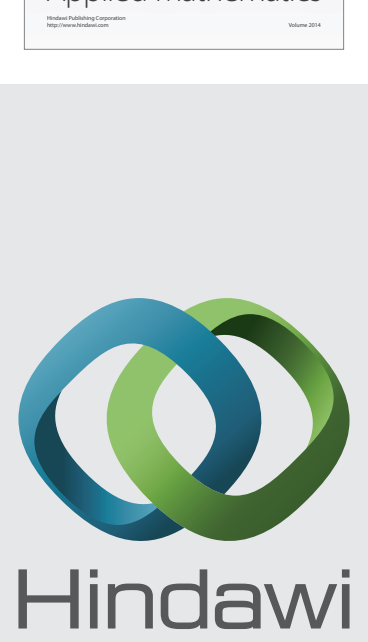

Submit your manuscripts at http://www.hindawi.com
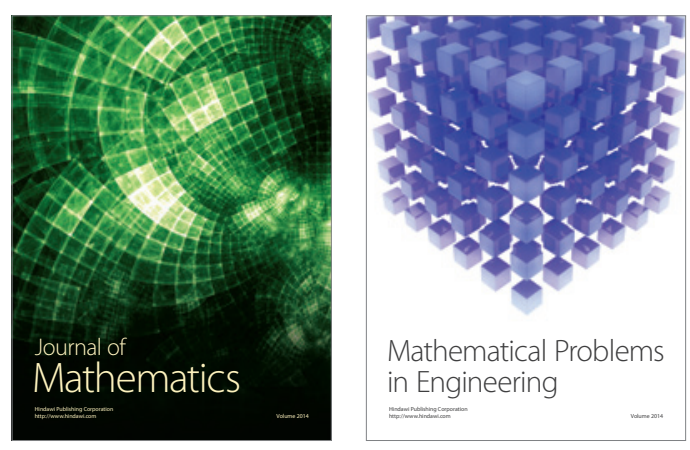

Mathematical Problems in Engineering
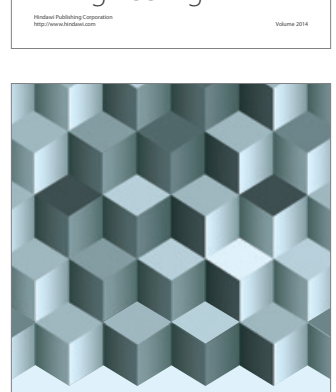

Journal of

Function Spaces
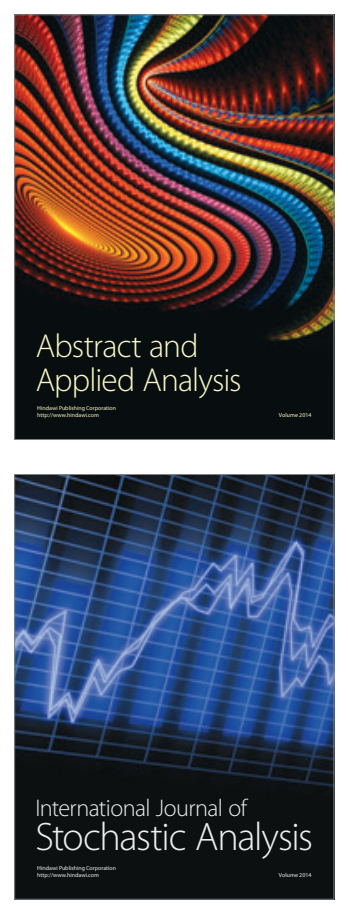

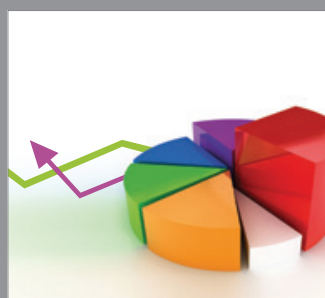

ournal of

Probability and Statistics

Promensencen
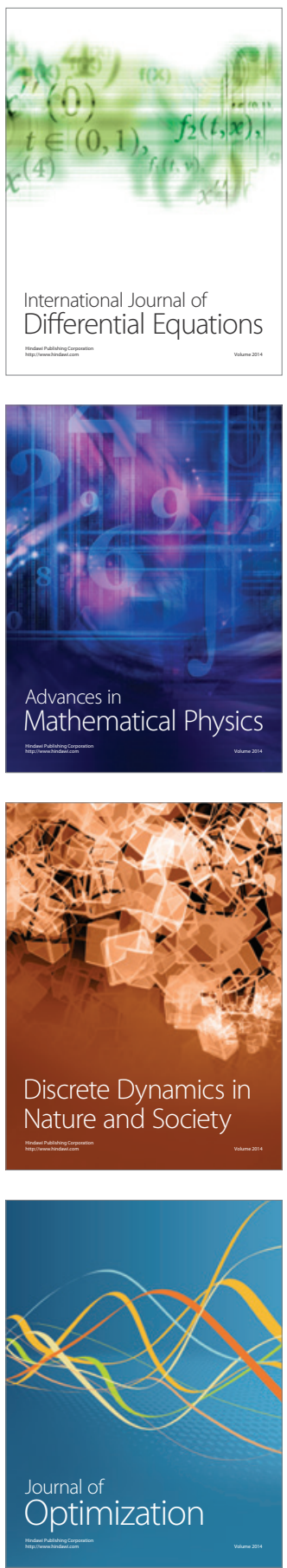